\title{
ENSAT registry-based randomized clinical trials for adrenocortical carcinoma
}

\author{
Joakim Crona', Eric Baudin², Massimo Terzolo ${ }^{3}$, Alexandra Chrisoulidou ${ }^{4}$, Anna Angelousi ${ }^{5}$, Cristina L Ronchi6,7, \\ Cristina Lamas Oliveira ${ }^{8}$, Els J M Nieveen van Dijkum ${ }^{9}$, Filippo Ceccato ${ }^{10}$, Françoise Borson-Chazot ${ }^{11}$, \\ Giuseppe Reimondo ${ }^{12}$, Guido A M Tiberi ${ }^{13}$, Hester Ettaieb ${ }^{14}$, Andreas Kiriakopoulos ${ }^{15}$, Letizia Canu ${ }^{16}$, \\ Darko Kastelan ${ }^{17}$, Esthr Osher ${ }^{18}$, Eugenia Yiannakopoulou ${ }^{19}$, Giorgio Arnaldi ${ }^{20}$, Guillaume Assié21,22, \\ Isabel Paiva ${ }^{23}$, Isabelle Bourdeau ${ }^{24}$, John Newell-Price ${ }^{25}$, Karolina M Nowak ${ }^{26}$, M Tous Romero ${ }^{27}$, \\ Maria Cristina De Martino ${ }^{28}$, Maria João Bugalho ${ }^{29}$, Mark Sherlock ${ }^{30}$, Marie-Christine Vantyghem ${ }^{31}$, \\ Michael Conall Dennedy ${ }^{32}$, Paula Loli ${ }^{33}$, Patrice Rodien ${ }^{34}$, Richard Feelders ${ }^{35}$, Ronald de Krijger ${ }^{36,37}$, \\ Sam Van Slycke ${ }^{38}$, Simon Aylwin ${ }^{39}$, Valentina Morelli ${ }^{40}$, Laurent Vroonen ${ }^{41}$, Zulfiya Shafigullina ${ }^{42}$, Irina Bancos ${ }^{43}$, \\ Małgorzata Trofimiuk-Müldner ${ }^{44}$, Marcus Quinkler ${ }^{45}$, Michaela Luconi ${ }^{16}$, Matthias Kroiss ${ }^{7,46}$, \\ Mitsuhide Naruse ${ }^{47}$, Peter Igaz ${ }^{48,49}$, Radu Mihai ${ }^{50}$, Silvia Della Casa ${ }^{51}$, Alfredo Berruti ${ }^{52}$, Martin Fassnacht ${ }^{7,46}$ and \\ Felix Beuschlein 53,54
}

${ }^{1}$ Department of Medical Sciences, Uppsala University, Uppsala, Sweden, ${ }^{2}$ Gustave Roussy, Université Paris-Saclay, Département d'imagerie, Service d'oncologie endocrinienne, Villejuif, France, ${ }^{3}$ Department of Clinical and Biological Sciences, University of Turin at San Luigi Hospital, Orbassano, Italy, ${ }^{2}$ Department of Endocrinology, Theagenio Cancer Hospital, Thessaloniki, Greece, ${ }^{5} 1$ st Department of Internal Medicine, Unit of Endocrinology, National and Kapodistrian University of Athens, Laiko Hospital, Athens, Greece, ${ }^{6}$ Institute of Metabolism and System Research, University of Birmingham, Birmingham, UK, ${ }^{7}$ Division of Endocrinology and Diabetes, University Hospital of Würzburg, Würzburg, Germany, ${ }^{8}$ Endocrinology Department, Complejo Hospitalario Universitario de Albacete, Albacete, Spain, ${ }^{9}$ Department of Surgery, Cancer Center Amsterdam, Amsterdam UMC, University of Amsterdam, Amsterdam, The Netherlands, ${ }^{10}$ Endocrinology Unit, Department of Medicine DIMED, University-Hospital of Padova, Padova, Italy, ${ }^{11}$ Hospices Civils de Lyon, Fédération d'Endocrinologie, Université Claude Bernard Lyon 1, Lyon, France, ${ }^{12}$ Internal Medicine, Department of Clinical and Biological Sciences, San Luigi Gonzaga Hospital, University of Turin, Orbassano, Turin, Italy, ${ }^{13}$ Surgical Clinic, Department of Clinical and Experimental Sciences, University of Brescia at ASST Spedali Civili, Brescia, Italy, ${ }^{14}$ Division of Endocrinology, Department of Internal Medicine, Maxima Medical Center, Eindhoven/Veldhoven, The Netherlands, ${ }^{15} 5$ th Surgical Clinic, 'Evgenidion Hospital' National and Kapodistrian University of Athens School of Medicine, Athens, Greece, ${ }^{16}$ Department of Experimental and Clinical Biomedical Sciences, University of Florence, Florence, Italy, ${ }^{17}$ Department of Endocrinology, University Hospital Centre Zagreb, Zagreb, Croatia, ${ }^{18}$ Institute of Endocrinology, Metabolism and Hypertension, Tel Aviv-Sourasky Medical Center Israel, Sackler Faculty of Medicine, Tel Aviv University, Tel Aviv, Israel, ${ }^{19}$ Department of Biomedical Sciences, Faculty of Health Sciences, University of West Attica, Athens, Greece, ${ }^{20}$ Division of Endocrinology, Department of Clinical and Molecular Sciences, Polytechnic University of Marche, Ancona, Italy, ${ }^{21}$ Université de Paris, Institut Cochin, INSERM, CNRS, Paris, France, ${ }^{22}$ Endocrinology, Assistance Publique Hôpitaux de Paris, Hôpital Cochin, Paris, France, ${ }^{23}$ Department os Endocrinology, Diabetes and Metabolism, Centro Hospitalar e Universitário de Coimbra, Coimbra, Portugal, ${ }^{24}$ Division of Endocrinology, Department of Medicine, Research Center, Centre Hospitalier de l'Université de Montréal (CHUM), Montreal, Québec, Canada, ${ }^{25}$ Department of Oncology and Metabolism, The Medical School University of Sheffield, Sheffield, UK, ${ }^{26}$ Department of Endocrinology, Centre of Postgraduate Medical Education, Bielanski Hospital, Warsaw, Poland, ${ }^{27}$ UGC Endocrinología y Nutrición, Hospital Universitario Virgen Macarena, Seville, Spain, ${ }^{28}$ Dipartimento di Medicina Clinica e Chirurgia, Sezione di Endocrinologia, Università Federico II di Napoli, Naples, Italy, ${ }^{29}$ Serviço de Endocrinologia, Diabetes e Metabolismo, CHULN and Faculdade de Medicina da Universidade de Lisboa, Lisboa, Portugal, ${ }^{30}$ Department of Endocrinology, Beaumont Hospital and the Royal College of Surgeons in Ireland, Dublin, Ireland, ${ }^{31}$ Endocrinology, Diabetology, Metabolism and Nutrition Department, Lille University Hospital, Lille, France, ${ }^{32}$ Department of Endocrinology \& Diabetes Mellitus, c/o Department of Medicine, Clinical Sciences Institute, National University of Ireland, Galway, Galway, Ireland, ${ }^{33}$ Ospedale Niguarda Ca' Granda, Endocrinology, Napoli, Italy, ${ }^{34}$ Service d'Endocrinologie Diabétologie et Nutrition, CHU d'Angers, Angers Cedex 9, France, ${ }^{35}$ Erasmus Medical Center, Division of Endocrinology, Department of Internal Medicine, Rotterdam, The Netherlands, ${ }^{36}$ Princess Máxima Center for Pediatric Oncology, Utrecht, The Netherlands , ${ }^{37}$ Department of Pathology, University Medical Center, Utrecht, The Netherlands, ${ }^{38}$ General and Endocrine Surgery, OLV Hospital Aalst, Aalst, Belgium, ${ }^{39}$ King's College Hospital, London, UK, ${ }^{40}$ Endocrinology Unit, Fondazione IRCCS Ca' Granda, Ospedale Maggiore Policlinico, Milan, Italy, ${ }^{41}$ Department of Endocrinology, Centre Hospitalier Universitaire de Liège, Liege, Belgium, ${ }^{42}$ Endocrinology Department, North-Western Medical University named after I.I.Mechnikov, Saint-Petersburg, Russia, ${ }^{43}$ Division of Endocrinology, Diabetes, Metabolism, and Nutrition, Mayo Clinic, Rochester, Minnesota, USA, ${ }^{44}$ Department of Endocrinology, Jagiellonian University Medical College, Kraków, Poland, ${ }^{45}$ Endocrinology in Charlottenburg, Berlin, Germany, ${ }^{46}$ Comprehensive Cancer Center Mainfranken, University of Würzburg, Würzburg, Germany, ${ }^{47}$ Endocrine Center, ljinkai Takeda General Hospital and Clinical Research Institute of Endocrinology and Metabolism, NHO Kyoto Medical Center, Kyoto, Japan, ${ }^{48}$ 2nd Department of Internal Medicine, Semmelweis University, Budapest, Hungary, ${ }^{49}$ MTA-SE Molecular Medicine Research Group, Hungarian Academy of Sciences and Semmelweis University, Budapest, Hungary, ${ }^{50}$ Churchill Cancer Centre, Department of Endocrine Surgery, Oxford University, Oxford, UK, ${ }^{51}$ Endocrinology Department, Gemelli Polyclinic Foundation, Catholic University, Rome, Italy, ${ }^{52}$ Department of Medical and Surgical Specialties, Radiological Sciences,

(C) 2021 European Society of Endocrinology Printed in Great Britain
Published by Bioscientifica Ltd. 
and Public Health, Medical Oncology, University of Brescia at ASST Spedali Civili, Brescia, Italy, ${ }^{53}$ Klinik für Endokrinologie, Diabetologie und Klinische Ernährung, Universitätsspital Zürich, Zürich, Switzerland, and ${ }^{54}$ Department of Endocrinology, Medizinische Klinik und Poliklinik IV, Klinikum der Universität München, Munich, Germany
Correspondence should be addressed to F Beuschlein Email

felix.beuschlein@usz.ch

\section{Abstract}

Adrenocortical carcinoma (ACC) is an orphan disease lacking effective systemic treatment options. The low incidence of the disease and high cost of clinical trials are major obstacles in the search for improved treatment strategies. As a novel approach, registry-based clinical trials have been introduced in clinical research, so allowing for significant cost reduction, but without compromising scientific benefit. Herein, we describe how the European Network for the Study of Adrenal Tumours (ENSAT) could transform its current registry into one fit for a clinical trial infrastructure. The rationale to perform randomized registry-based trials in ACC is outlined including an analysis of relevant limitations and challenges. We summarize a survey on this concept among ENSAT members who expressed a strong interest in the concept and rated its scientific potential as high. Legal aspects, including ethical approval of registry-based randomization were identified as potential obstacles. Finally, we describe three potential randomized registrybased clinical trials in an adjuvant setting and for advanced disease with a high potential to be executed within the framework of an advanced ENSAT registry. Thus we, therefore, provide the basis for future registry-based trials for ACC patients. This could ultimately provide proof-of-principle of how to perform more effective randomized trials for an orphan disease.

\section{Introduction}

Adrenocortical carcinoma (ACC) is a rare disease for which diagnostic approaches and therapeutic strategies have only gradually changed over the past decades $(1,2)$. Accordingly, the overall survival for patients diagnosed with ACC remains in the range of $3-4$ years $(3,4)$. Affected patients also experience severe morbidity due to endocrine disturbances as well as tumour growth (1, $5,6)$. In a recent review, we identified topics including disease prevention and earlier detection, improved riskstratification, controlling tumour growth and invasiveness as well as suppressing hormone production as unresolved problems that need to be addressed by research with the overarching aim to reduce ACC-related morbidity and mortality (1).

While clinical trials have the potential to explore strategies to approach these problems, the current research infrastructure fails in providing effective resources to perform such projects on rare diseases. In this context, registry-based clinical trials have emerged as a resourceefficient alternative solution to address clinical and translational research questions $(7,8,9,10)$. In this review, we aim to describe how registry-based trials could be used to advance care of patients with ACC. Furthermore, we argue that the European Network for the Study of Adrenal Tumours (ENSAT) is well-positioned to transform its current registry and advance its strong collaboration to implement registry-based clinical trials. Finally, we propose potential research projects with potential to be executed within this space.

\section{Randomized clinical trials}

A randomized controlled trial provides the experimental framework that aims to evaluate the effectiveness and safety of a medical intervention. By randomly assigning patients between experimental and control arms, it ensures the greatest reliability and validity of the results, by reducing impact from both known and unrecognized bias. Appropriately executed (11), it is considered as the gold standard for evaluating healthcare interventions. In contrast, those medical practices that are based on 
evidence from non-randomized controlled data are prone to bias and misinterpretation. Clinical trials lacking a control arm and those using historical controls have repeatedly been shown to exaggerate the efficacy of treatments (12). Similarly, early clinical trials on small or diverse patient samples are prone to find higher response rates than those in subsequent randomized studies (13). Overall, these shortcomings have well been exemplified in a systematic review of $>3000$ randomized clinical studies demonstrating that a total of 396 formerly established medical practices had been identified as lacking clinical benefit (14).

While there are a few reported randomized controlled trials on ACC (Table 1), patient scarcity and high resource demand has limited the use of this method. As a consequence, among the 25 recommendations with evidence rating in the recent ACC guidelines by the European Society for Endocrinology and ENSAT, none were considered to have strong underlying evidence and only three were graded as having moderate evidence (5). Currently, there is only one randomized clinical trial active within the space of ACC: Mitotane With or Without Cisplatin and Etoposide After Surgery in Treating Participants With Stage I-III ACC With High Risk of Recurrence (ADIUVO-2, NCT03583710). This study has been designed to be executed within a clinical environment but has yet to be directly integrated into an established patient registry. This sets the stage for further optimization of the clinical trial method for the study of ACC in order to further improve clinical evidence and refine patient care.

\section{Registry-based clinical trials}

A randomized registry-based clinical trial is a prospective study using a clinical registry for patient identification, trial conduct and outcomes reporting. The registrybased randomized clinical trial maintains the strengths of a prospective clinical study, including high internal validity, stringent patient stratification, randomization to ensure unbiased study of interventions and analysing patient outcomes to determine the effect of the studied intervention (Table 2) $(7,9,15)$. In variance to conventional trials, registry-based studies provide the opportunity to lower costs and ensure more rapid patient inclusion $(7,9,15)$. This method is particularly suitable for evaluation of interventions that are already established within the field, with documented data on adverse events and that does not require additional
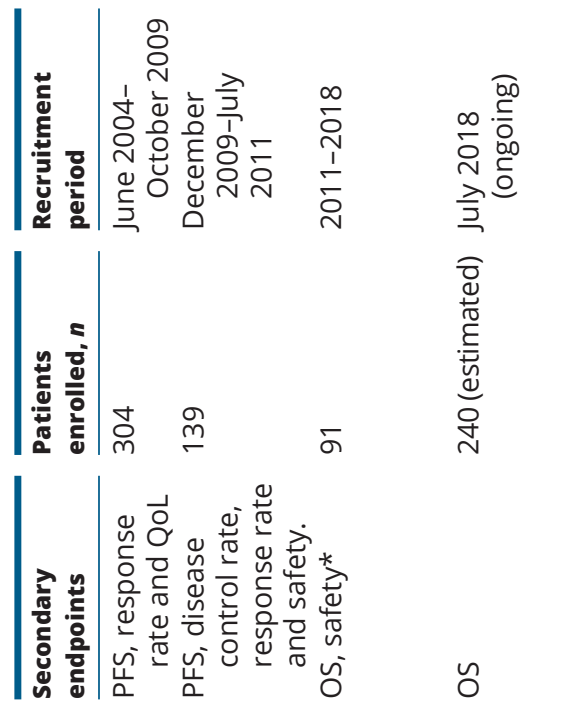

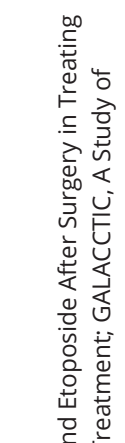
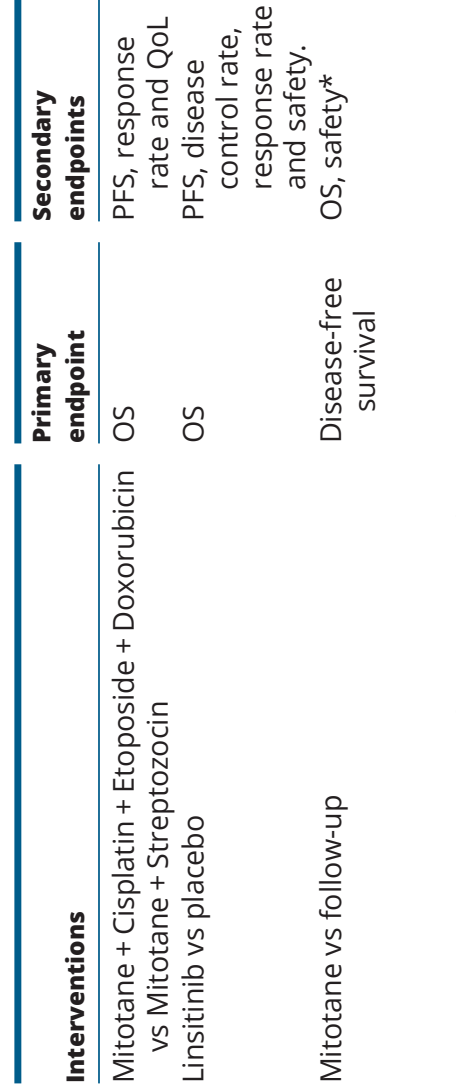

ัั

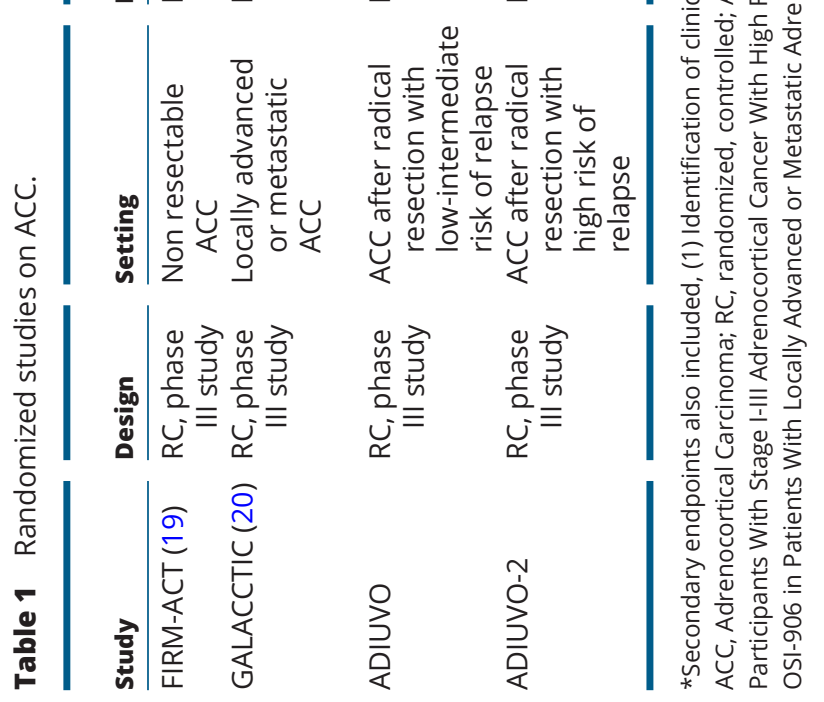

https://eje.bioscientifica.com 
Table 2 Comparison between conventional and registry-based clinical trials.

\begin{tabular}{ll}
\hline Scientific aspects & \multicolumn{1}{c}{ Conventional randomized clinical trials } \\
\cline { 2 - 3 } & Typically investigates new interventions \\
& Capacity to include complex criteria and \\
& outcome measures including adverse \\
events & Highly selected patient populations \\
Ensured high validity and data quality \\
through monitoring \\
High cost \\
Executed within a dedicated environment \\
separate to standard clinical care \\
Individual identification of suitable \\
candidates \\
Demanding to achieve fast patient inclusion \\
Regulatory environment adopted to \\
conventional trials
\end{tabular}

\begin{tabular}{l} 
Registry-based randomized clinical trials \\
\hline Typically investigates established interventions with \\
documented safety profiles \\
Patient criteria and outcome factors less detailed \\
and limited to existing registry \\
Broad and representative patient populations \\
Validity and data quality depending on registry \\
quality \\
Low cost \\
Uses established clinical infrastructure. Integration \\
with existing high quality registries mandatory. \\
Registry may facilitate integrated identification of \\
suitable patients \\
More rapid patient inclusion rate due to broad \\
eligibility and wide dissemination of centers \\
Regulatory environment not adopted to the \\
registry-based clinical trial
\end{tabular}

evaluations than those already performed within standard clinical practice.

A registry structure can be used to identify eligible patients, randomize between different interventions, provide follow-up data and evaluate outcomes. To remain resource-effective, addition of procedures beyond standard clinical practice should be avoided. Experience from cardiovascular research has demonstrated that registry-based clinical studies can be performed with more than $90 \%$ cost-saving compared to conventional trials $(7,9)$. Ongoing developments of this method include refinements of both biostatistical analysis and interpretation (16).

To our knowledge, there are no reported registrybased clinical trials and only a few ongoing within the field of medical oncology or endocrinology. In a review by Foroughi and colleagues, ongoing registry-based clinical trials were described (9) from which we select two relevant examples:

ALT-TRACC (17) is a phase II clinical trial randomizing patients with treatment naïve metastatic colorectal cancer between alternating oxaliplatin and irinotecan doublet schedules (experimental arm) vs continuous doublet chemotherapy (control arm). Primary objective is to evaluate the feasibility of conducting a multi-center, prospective, registry-based randomized clinical trial. The primary endpoint is recruitment rate. Secondary objectives focus on both efficacy and toxicity by collecting data from medical records and other data collection tools. The aim is to estimate progression-free survival and radiological response rates. The study is based on the 'Treatment of Recurrent and Advanced Colorectal Cancer' registry (9).
EX-TEM (18) is a phase III trial randomizing patients with newly diagnosed glioblastoma to 6 (control arm) vs 12 (experimental arm) cycles of post-radiation temozolomide chemotherapy. The primary objective is to study treatment efficacy and the primary endpoint is overall survival. Secondary endpoints include adverse events and the necessity for temozolomide dose modification determined by data recorded in the medical records. The study makes usage of the 'Brain Registry Australia: Innovation and Translation' registry (9).

\section{Current and previous randomized trials for ACC}

An overview of randomized clinical studies on ACC is provided in Table 1. FIRM-ACT was the first randomized study performed on ACC and compared the efficacy of a chemotherapy combination (etoposide, cisplatin and doxorubicin, EDP) plus mitotane vs streptozocin plus mitotane in the advanced setting (19). It reported a hazard ratio of 0.55 (95\% CI: 0.43-0.69) in favour for EDP plus mitotane for progression-free survival. Survival was not significantly different, hazard ratio 0.79 (95\% CI: 0.61-1.02) in favour for EDP + mitotane. Quality of Life according to the EORTC QLQ-C30 questionnaire revealed no changes at follow-up compared to baseline for the two treatment arms.

As recruitment within the FIRM-ACT protocol had been achieved, it was quickly followed by GALACCTIC, a randomized phase III trial of linsitinib (inhibitor of IGF-1R and the insulin receptor) vs placebo for locally advanced 
or metastatic ACC (20). No difference in overall survival between linsitinib and placebo was noted, HR 0.94 (95\% CI: 0.61-1.44). This study did, however, provide valuable information on the behaviour of untreated metastatic ACC progressive after mitotane therapy. In the control arm, median survival was 356 days (95\% CI 249-556) while the disease control rate was $34.7 \%$ (95\% CI: $21.7-49.6$ ) at 6 weeks and $8.2 \%$ at 12 weeks.

There are currently two ongoing randomized trials on ACC, both in the adjuvant setting (Table 1), mitotane vs follow-up in low to intermediate risk ACC (ADIUVO study) and mitotane vs mitotane plus cisplatin and etoposide (ADIUVO-2 study) in high-risk ACC. These trials will be fundamental to evaluate current practices for adjuvant therapy that are currently supported by retrospective data (21).

\section{The European network for the study of adrenal tumours}

The European Network for the Study of Adrenal Tumours was formally established in 2002 through a merger of three already existing national networks on adrenal research: COMETE in France, GANIMED in Germany, and NISGAT in Italy with further teams joining in from the United Kingdom. In 2009, ENSAT became a membership-based society with statutes and by-laws. An increasing number of researchers and health workers have joined in the efforts of the ENSAT with currently 479 members from 35 different countries. The European Network for the Study of Adrenal Tumours has structured its operation under four different working groups by disease subtype: ACC, pheochromocytoma and paraganglioma, aldosteroneproducing adenoma and non-aldosterone producing cortical adrenal adenomas. Through its patient registry, the largest body of clinical annotations and biospecimens from patients with adrenal tumours has been aggregated (22). Currently (April 2020) it includes data from 17680 patients of 107 institutions, representing 33 predominantly European countries. A long list of disease-specific clinical annotations has been collected. Current limitations of the ENSAT registry include its non-consecutive patient enrolment and lack of quality control.

Based on the information reviewed in previous sections, we hypothesized that registry-based trials would be a potential new tool to allow for more efficient studies on adrenal tumours. We hypothesized further that ENSAT would be ideally positioned to implement this technology as it already forms a strong network with large patient populations and operates a prospective registry. Finally, ACC was identified as the most suitable patient population among adrenal tumours to be evaluated in a pilot project due to large unmet needs in combination with up-to-date clinical practice guidelines and potentially relevant study endpoint already available in the registry $(5,23)$.

\section{The ENSAT ACC registry}

Currently, the ACC database includes data from 3,835 patients from 63 institutions (April 2020). It is structured under the following sections; diagnostic procedures (34 variables), tumour staging (16 variables), biomaterial (20 variables), chemoembolization (four variables), chemotherapy (7 variables), follow-up (18 variables), metabolomics (2 variables), mitotane (nine variables), pathology (20 variables), radiofrequency (5 variables), radiotherapy (8 variables) and surgery (6 variables). In total, these comprehensive data can be used to study endpoints relevant for patients with ACC; overall survival, recurrence-free survival, progression-free survival (accordingly to local analysis, but no specific protocol for radiological evaluation is requested, yet) and early discontinuation of medical therapy. Furthermore, appropriate factors for disease characterization can be used as inclusion/exclusion criteria as well as being incorporated into a future randomization module.

\section{Assessing the potential for registry-based clinical trials within ENSAT}

Two online surveys as well as discussions at scientific meetings had been conducted to determine the potential of registry-based clinical trials to be performed within the ENSAT community (Supplementary materials and methods, see section on supplementary materials given at the end of this article). In a first survey (Supplementary Table 1) responses had been collected from 86 members, including 66 full members and 20 associate members. The respondents represented 22 different countries; Italy $(n=22)$, Germany $(n=10)$, France $(n=8)$, Greece $(n=7)$, United Kingdom $(n=7)$, Netherlands $(n=6)$ and Spain $(n=4)$ being the most frequent. The high interest for registry-based clinical trials in ACC was reflected not only in the active participation in online surveys and real-life meetings, but was also expressed directly in the surveys 
through the rated scientific potential, mean score 4.5 (maximum 5), and the anticipation to collaborate and contribute, with a mean rating of 4.3 (maximum 5).

General topics for ENSAT registry-based trials were proposed with positive/negative response options available (Fig. 1A); evaluation of drugs or other medical interventions (90\% positive), evaluation of prognostic or predictive biomarkers for therapeutic stratification (89\% positive), prospective collection of clinical data and/or bio samples (71\% positive), and comparison of different follow-up strategies (69\% positive). Study participants were also asked, which ACC patient population should primarily be addressed (positive/negative response options available); neo-adjuvant setting (75\% positive), adjuvant setting ( $87 \%$ positive) and advanced disease (61\% positive). Next, survey participants were asked if they would foresee legal or any other administrative challenges related to registry-based clinical trials, which was answered with yes in $56 \%$ of cases with eight free text comments provided. Among these responses, reluctance from ethical review boards to provide ethical permissions were specifically mentioned. Another example demonstrating the strong

A Survey 1, Question 3: In your opinion what general topics should be targeted by an ENSAT registry-based trial in ACC?

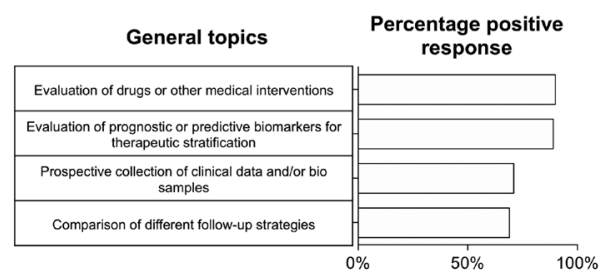

B

Survey 2, Question 3: Please rank the following potential projects on ACC accordingly to your priority, $6=$ maximum priority

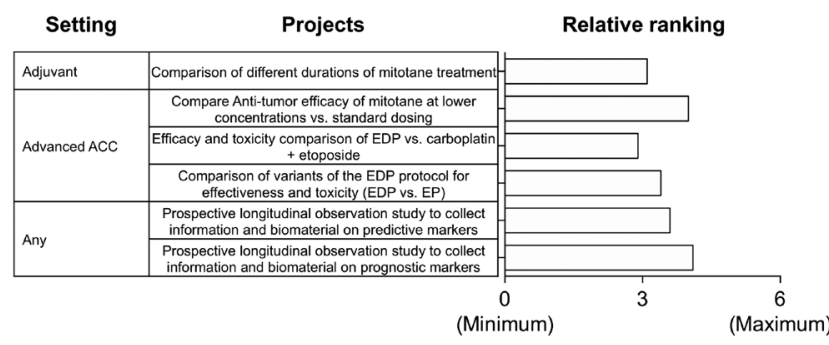

\section{Figure 1}

(A and B) Selected data from surveys 1 and 2 demonstrating different research topics where a registry-based trial infrastructure was proposed to provide value to patients with adrenocortical carcinoma. ACC, Adrenocortical carcinoma; EDP, Etoposide + doxorubicin + platinum-based chemotherapy; $E P$, etoposide + platinum-based chemotherapy. interest in registry-based clinical trials could be noted in the active discussion of particular scientific projects: There were a total of 48 different research projects being proposed. The ENSAT ACC working group scientific board prioritized these projects based on scientific quality and feasibility for further evaluation.

In the subsequent survey (Supplementary Table 2) there were 62 respondents, 50 full members and 12 associate members. These represented 19 different countries with Italy $(n=19)$, France $(n=6)$, Germany $(n=6)$, Greece $(n=5)$ and the United Kingdom $(n=5)$ as the most frequent. A total of $87 \%$ of responders phrased the expectation that a registry-based clinical trial would be accepted by the local ethical committee, with eight additional comments in free text. In the next question, $43 \%$ assumed that randomization of study sites to different interventions ('cluster randomization') would be more likely to be acceptable to ethical review boards compared to randomization of individual patients. Furthermore, concrete ideas for problems to be addressed within an ENSAT registry-based platform were collected.

\section{Proposal for registry-based studies on ACC based on the ENSAT platform}

Of the 48 different research projects being proposed by the ENSAT ACC working group, the scientific board and its members had previously selected and prioritized the following projects that gained particularly high scoring based on scientific value and feasibility (Fig. 1B):

\section{Adjuvant setting}

Comparison of different durations of mitotane treatment for effectiveness and toxicity: Adjuvant treatment with mitotane is recommended in patients without macroscopic residual tumour after surgery who have a perceived high risk of recurrence $(5,21,24)$. However, the optimal duration of mitotane treatment, to balance efficacy and adverse effects of the compound is currently unknown. Therefore, a randomized controlled study between a duration of for example, 2 vs 5 years of mitotane treatment would be informative.

\section{Advanced ACC I}

Comparison of different first-line chemotherapy protocols for effectiveness and toxicity: The most validated first-line treatment option for unresectable and advanced ACC is 
the combination of etoposide, doxorubicin, cisplatin, and mitotane (EDP-M) (19). Treatment with EDP-M comes with a risk of adverse events. Based on small trials $(25,26)$ and individual experience (5) it has been hypothesized that omitting doxorubicin from the treatment protocol would increase tolerability without a clinically relevant loss of efficacy. This hypothesis could be evaluated through a randomized controlled study between EDP-M (standard arm) vs etoposide, cisplatin and mitotane (experimental arm).

\section{Advanced ACC II}

Comparison of anti-tumour efficacy of mitotane at different concentrations: It is believed that mitotane toxicity and efficacy is strongly correlated to plasma levels of the compound $(27,28,29)$. It has been hypothesized that by lowering the therapeutic concentration target for mitotane in advanced ACC, patients would experience less treatment related toxicity. This could potentially result in improved quality of life without clinically significant loss in efficacy. For this purpose, a randomized controlled study between standard therapy aiming at a mitotane blood level $>14 \mathrm{mg} / \mathrm{L}$ (standard arm) vs a mitotane regime aiming at lower concentration (e.g. $>10 \mathrm{mg} / \mathrm{L}$; experimental arm) would lead to clinically important information.

Potential objectives to be investigated in these three proposals include the evaluation of recruitment feasibility, quality of data capture, patient benefit in terms of overall survival and quality of life as well as drug tolerability.

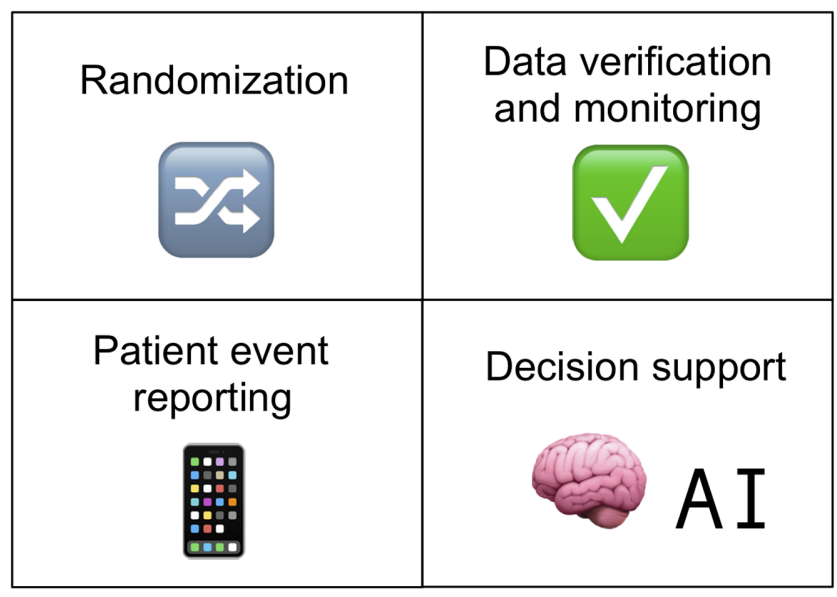

Figure 2

Unmet needs of the European Network for the Study of Adrenal Tumours database in order to advance research and perform efficient randomized registry-based trials.
Furthermore, we propose that quality of life could be measured through patient self-reporting through a webbased application (currently not available in the ENSAT registry). In addition, safety could be described from the documentations made in the patient records. We also argue that both cluster and patient randomization could be used to address these three research questions. The studies could also be designed as superiority and/or nonsuperiority trials, all depending on what primary endpoint is finally selected.

In addition to studying different interventions, we envision the possibility to execute prospective longitudinal observation studies to collect information and biomaterial on predictive markers of treatment response as well as prognostic factors. The underlying rationale comes from the rapid advances in our understanding of the biology of ACC $(1,30,31,32,33,34,35)$, which translates into a need for efficient test beds to evaluate new biomarkers for different clinical purposes.

\section{Challenges}

Examples in clinical cardiovascular research has provided a clear proof of concept of how a strong network can be enhanced to perform registry-based clinical trials through technical advances of the current infrastructure but only minor changes in clinical and research practice (7). One potential factor limiting the dissemination of registrybased trials could be legal and administrative restrictions. In our surveys, a high proportion of participants (87\%) anticipated acceptance of a registry-based trial by their local ethical review boards. However, as the registrybased randomized trial is a concept currently lacking a clear definition it is expected to be treated with the same level of scrutiny as conventional clinical trials. This will impose rules and regulations not applicable to registrybased randomized trials. As such, the current legislative environment needs to be adopted to fit randomized registry-based clinical trials in order to ensure a lower complexity that will otherwise increase costs.

While the foundation for a future infrastructure for registry-based trails exist within ENSAT, additional method development will be required including a randomization module as well as the possibility for source data verification (Fig. 2). There is also a need for data monitoring to ensure high validity of the data within the registry. Furthermore, our work also raised the potential to implement clinical decision support, active suggestion of potential research studies and integration with patient self-reporting into 
the ENSAT registry. While such infrastructure upgrades are all technically feasible, additional resources will be necessary for its implementation. And as this research direction is pursued in other medical settings, ENSAT could potentially co-operate with other relevant registries for method development and to share experiences.

\section{Summary}

The ENSAT ACC community has expressed a strong interest and support in registry-based trials as a new infrastructure with potential to significantly advance care for patients with this rare disease. This review summarises the scientific foundation for this research direction and outlines potential questions to be addressed within such a new infrastructure and provides a roadmap for future pilot projects.

\section{Supplementary materials}

This is linked to the online version of the paper at https://doi.org/10.1530/ EJE-20-0800.

\section{Declaration of interest}

J C received lecture honoraria from Novartis and educational honoraria from NET connect (funded by Ipsen). M K received institutional funding for a clinical trial of cabozantinib in advanced adrenocortical carcinoma from Ipsen. I B reports advisory board participation with Corcept and HRA Pharma and consultancy fee with ClinCore outside the submitted work. F B $C$ received honoraria for lecture or consultancy work from Ipsen, Novartis, Bayer, Esai and Novo Nordisk (unrelated to the present topic). S A has received lecture honoraria from HRA pharma. J N P has received research and consultancy grants from HRA Pharma, Novartis, Diurnal, Recordati (unrelated to the present topic). M N received lecture honoraria from DAIICHI SANKYO Company, Ltd., and Kyowaki rin Co.Ltd. and consultancy grants from Ono Pharmaceutical Co. Ltd., and FUJIF ILM RI Pharma Co.,Ltd. (unrelated to the present topic). Guillaume Assie and Martin Fassnacht are Associate Editors of European Journal of Endocrinology. They were not involved in the editorial or peer review process of this paper, on which they are listed as authors.

\section{Funding}

J $C$ received funding from Region Uppsala, Cancerfonden and Tore Nilsons Stiftelse, F B and M F were supported by the German Research Foundation (DFG) project number 314061271 (CRC/TRR 205). M N received funding from Japan Agency for Medical Research and Development (AMED) (JP19ek0109352).

\section{Acknowledgements}

We thank Drs Ivana Kraljevic, Jérôme Bertherat, Christina Pamporaki, Timo Deutschbein, Altieri Barbara, Gregory Kaltsas, Dimitra A Vassiliadi, Boudina Maria, Vincent Montauban, Massimo Mannelli, Gabriella Nesi, Giulia Cantini, Mirko Parasiliti Caprino, Masakatsu Sone, Rebecca Steenaard and Wiebke Arlt for contributing to this project by taking any of the online surveys. The authors also thank Dr Silviu Sbiera for excellent technical support.

\section{References}

1 Crona J \& Beuschlein F. Adrenocortical carcinoma - towards genomics guided clinical care. Nature Reviews: Endocrinology 201915 548-560. (https://doi.org/10.1038/s41574-019-0221-7)

2 Else T, Kim AC, Sabolch A, Raymond VM, Kandathil A, Caoili EM, Jolly S, Miller BS, Giordano TJ \& Hammer GD. Adrenocortical carcinoma. Endocrine Reviews 201435 282-326. (https://doi. org/10.1210/er.2013-1029)

3 Kerkhofs TM, Verhoeven RH, Van der Zwan JM, Dieleman J, Kerstens MN, Links TP, Van de Poll-Franse LV \& Haak HR. Adrenocortical carcinoma: a population-based study on incidence and survival in the Netherlands since 1993. European Journal of Cancer 201349 2579-2586. (https://doi.org/10.1016/j. ejca.2013.02.034)

4 Tella SH, Kommalapati A, Yaturu S \& Kebebew E. Predictors of survival in adrenocortical carcinoma: an analysis from the National Cancer Database. Journal of Clinical Endocrinology and Metabolism 2018103 3566-3573. (https://doi.org/10.1210/jc.2018-00918)

5 Fassnacht M, Dekkers OM, Else T, Baudin E, Berruti A, de Krijger R, Haak HR, Mihai R, Assie G \& Terzolo M. European Society of Endocrinology Clinical Practice Guidelines on the management of adrenocortical carcinoma in adults, in collaboration with the European Network for the Study of Adrenal Tumors. European Journal of Endocrinology 2018179 G1-G46. (https://doi.org/10.1530/EJE-180608)

6 Steenaard RV, Kremers MNT, Michon LA, Zijlstra M \& Haak HR. Patient and partner perspectives on health-related quality of life in adrenocortical carcinoma. Journal of the Endocrine Society 20204 bvaa040. (https://doi.org/10.1210/jendso/bvaa040)

7 James S, Rao SV \& Granger CB. Registry-based randomized clinical trials - a new clinical trial paradigm. Nature Reviews: Cardiology 2015 12 312-316. (https://doi.org/10.1038/nrcardio.2015.33)

8 Wallentin L, Gale CP, Maggioni A, Bardinet I \& Casadei B. EuroHeart: European unified registries on heart care evaluation and randomized trials. European Heart Journal 201940 2745-2749. (https://doi. org/10.1093/eurheartj/ehz599)

9 Foroughi S, Wong HL, Gately L, Lee M, Simons K, Tie J, Wilks Burgess AW \& Gibbs P. Re-inventing the randomized controlled trial in medical oncology: the registry-based trial. Asia-Pacific Journal of Clinical Oncology 201814 365-373. (https://doi.org/10.1111/ ajco.12992)

10 Lauer MS \& D'Agostino Sr RB. The randomized registry trial - the next disruptive technology in clinical research? New England Journal of Medicine 2013369 1579-1581. (https://doi.org/10.1056/ NEJMp1310102)

11 Schulz KF, Altman DG, Moher D \& CONSORT. CONSORT 2010 Statement: updated guidelines for reporting parallel group randomised trials. BMJ 2010340 c332. (https://doi.org/10.1136/bmj.c332)

12 Sacks H, Chalmers TC \& Smith H, Jr. Randomized versus historical controls for clinical trials. American Journal of Medicine 198272 233-240. (https://doi.org/10.1016/0002-9343(82)90815-4)

13 Zia MI, Siu LL, Pond GR \& Chen EX. Comparison of outcomes of phase II studies and subsequent randomized control studies using identical chemotherapeutic regimens. Journal of Clinical Oncology 200523 6982-6991. (https://doi.org/10.1200/JCO.2005.06.679)

14 Herrera-Perez D, Haslam A, Crain T, Gill J, Livingston C, Kaestner V, Hayes M, Morgan D, Cifu AS \& Prasad V. A comprehensive review of randomized clinical trials in three medical journals reveals 396 medical reversals. eLife 20198 e45183. (https://doi.org/10.7554/ eLife.45183) 
15 Uppsala Clinical Research Center. https://www.ucr.uu.se/en/services/ r-rct. Accessed on 2020-03-09.

16 Nyberg K \& Hedman P. Swedish guidelines for registry-based randomized clinical trials. Upsala Journal of Medical Sciences $2019 \mathbf{1 2 4}$ 33-36. (https://doi.org/10.1080/03009734.2018.1550453)

17 ALT-TRACC Study. http://www.anzctr.org.au/Trial/Registration/ TrialReview.aspx?ACTRN=12618001480279. Accessed on 2020-05-01.

18 EX-TEM Study. http://www.anzctr.org.au/Trial/Registration/ TrialReview.aspx?id=376473\&isReview=true. Accessed on 2020-05-01.

19 Fassnacht M, Terzolo M, Allolio B, Baudin E, Haak H, Berruti A, Welin S, Schade-Brittinger C, Lacroix A, Jarzab B et al. Combination chemotherapy in advanced adrenocortical carcinoma. New England Journal of Medicine 2012366 2189-2197. (https://doi.org/10.1056/ NEJMoa1200966)

20 Fassnacht M, Berruti A, Baudin E, Demeure MJ, Gilbert J, Haak H, Kroiss M, Quinn DI, Hesseltine E, Ronchi CL et al. Linsitinib (OSI906) versus placebo for patients with locally advanced or metastatic adrenocortical carcinoma: a double-blind, randomised, phase 3 study. Lancet: Oncology 201516 426-435. (https://doi.org/10.1016/ S1470-2045(15)70081-1)

21 Stell A \& Sinnott R. The ENSAT registry: a digital repository supporting adrenal cancer research. Studies in Health Technology and Informatics 2012178 207-212.

22 Fassnacht M, Arlt W, Bancos I, Dralle H, Newell-Price J, Sahdev A, Tabarin A, Terzolo M, Tsagarakis S \& Dekkers OM. Management of adrenal incidentalomas: European Society of Endocrinology Clinical Practice Guideline in collaboration with the European Network for the Study of Adrenal Tumors. European Journal of Endocrinology 2016 175 G1-G34. (https://doi.org/10.1530/EJE-16-0467)

23 Terzolo M, Angeli A, Fassnacht M, Daffara F, Tauchmanova L, Conton PA, Rossetto R, Buci L, Sperone P, Grossrubatscher E et al. Adjuvant mitotane treatment for adrenocortical carcinoma. New England Journal of Medicine 2007356 2372-2380. (https://doi. org/10.1056/NEJMoa063360)

24 Berruti A, Grisanti S, Pulzer A, Claps M, Daffara F, Loli P, Mannelli M, Boscaro M, Arvat E, Tiberio G et al. Long-term outcomes of adjuvant mitotane therapy in patients with radically resected adrenocortical carcinoma. Journal of Clinical Endocrinology and Metabolism 2017102 1358-1365. (https://doi.org/10.1210/ jc.2016-2894)

25 Bonacci R, Gigliotti A, Baudin E, Wion-Barbot N, Emy P, Bonnay M, Cailleux AF, Nakib I, Schlumberger M \& Réseau Comète. Cytotoxic therapy with etoposide and cisplatin in advanced adrenocortical carcinoma. British Journal of Cancer 199878 546-549. (https://doi. org/10.1038/bjc.1998.530)

26 Williamson SK, Lew D, Miller GJ, Balcerzak SP, Baker LH \& Crawford ED. Phase II evaluation of cisplatin and etoposide followed by mitotane at disease progression in patients with locally advanced or metastatic adrenocortical carcinoma: a Southwest Oncology
Group Study. Cancer 200088 1159-1165. (https://doi.org/10.1002/ (SICI)1097-0142(20000301)88:5<1159::AID-CNCR28>3.0.CO;2-R)

27 Megerle F, Herrmann W, Schloetelburg W, Ronchi CL, Pulzer A, Quinkler M, Beuschlein F, Hahner S, Kroiss M, Fassnacht M et al. Mitotane monotherapy in patients with advanced adrenocortical carcinoma. Journal of Clinical Endocrinology and Metabolism 2018103 1686-1695. (https://doi.org/10.1210/jc.2017-02591)

28 Hermsen IG, Fassnacht M, Terzolo M, Houterman S, den Hartigh J, Leboulleux S, Daffara F, Berruti A, Chadarevian R, Schlumberger M et al. Plasma concentrations of o, $\mathrm{p}^{\prime} \mathrm{DDD}, \mathrm{o}, \mathrm{p}^{\prime} \mathrm{DDA}$, and o, $\mathrm{p}^{\prime} \mathrm{DDE}$ as predictors of tumor response to mitotane in adrenocortical carcinoma: results of a retrospective ENS@T multicenter study. Journal of Clinical Endocrinology and Metabolism 201196 1844-1851. (https://doi.org/10.1210/jc.2010-2676)

29 Baudin E, Pellegriti G, Bonnay M, Penfornis A, Laplanche A, Vassal G \& Schlumberger M. Impact of monitoring plasma 1,1-dichlorodiphenildichloroethane (o,p'DDD) levels on the treatment of patients with adrenocortical carcinoma. Cancer 200192 1385-1392. (https://doi.org/10.1002/10970142(20010915)92:6<1385::aid-cncr1461>3.0.co;2-2)

30 Assie G, Jouinot A, Fassnacht M, Libe R, Garinet S, Jacob L, Hamzaoui N, Neou M, Sakat J, de La Villéon B et al. Value of molecular classification for prognostic assessment of adrenocortical carcinoma. JAMA Oncology 2019. (https://doi.org/10.1001/ jamaoncol.2019.1558)

31 Assie G, Letouze E, Fassnacht M, Jouinot A, Luscap W, Barreau O, Omeiri H, Rodriguez S, Perlemoine K, René-Corail F et al. Integrated genomic characterization of adrenocortical carcinoma. Nature Genetics 201446 607-612. (https://doi.org/10.1038/ng.2953)

32 Schweitzer S, Kunz M, Kurlbaum M, Vey J, Kendl S, Deutschbein T, Hahner S, Fassnacht M, Dandekar T \& Kroiss M. Plasma steroid metabolome profiling for the diagnosis of adrenocortical carcinoma. European Journal of Endocrinology 2019180 117-125. (https://doi. org/10.1530/EJE-18-0782)

33 Gara SK, Lack J, Zhang L, Harris E, Cam M \& Kebebew E. Metastatic adrenocortical carcinoma displays higher mutation rate and tumor heterogeneity than primary tumors. Nature Communications 20189 4172. (https://doi.org/10.1038/s41467-018-06366-z)

34 Lippert J, Appenzeller S, Liang R, Sbiera S, Kircher S, Altieri B, Nanda I, Weigand I, Gehrig A, Steinhauer S et al. Targeted molecular analysis in adrenocortical carcinomas: a strategy toward improved personalized prognostication. Journal of Clinical Endocrinology and Metabolism 2018 103 4511-4523. (https://doi.org/10.1210/jc.2018-01348)

35 Mohan DR, Lerario AM, Else T, Mukherjee B, Almeida MQ Vinco M, Rege J, Mariani BMP, Zerbini MCN, Mendonca BB et al. Targeted assessment of GOS2 methylation identifies a rapidly recurrent, routinely fatal molecular subtype of adrenocortical carcinoma. Clinical Cancer Research 201925 3276-3288. (https://doi. org/10.1158/1078-0432.CCR-18-2693)

Received 13 July 2020

Revised version received 15 October 2020

Accepted 5 November 2020 